\title{
Thermodynamic Stability for Materials with Substructure
}

\author{
P.M. Mariano \\ DICeA, University of Florence, Firenze, Italy \\ paolo.mariano@unifi.it
}

\section{INTRODUCTION}

Connections between thermodynamics and stability for simple continuous bodies have been largely discussed in the scientific literature /1-5/. However, a few attempts (see, e.g., /6-8/) have been developed to analyze the same issues in complex bodies where the material substructure has a prominent influence on the gross behavior. The presence of material substructure may, in fact, generate possible local material instabilities and bifurcations (see /7/ for the special case of nematic liquid crystals). Moreover, it influences even the global stability criteria of motion. We collect here some preliminary issues towards the study of thermodynamic stability of complex bodies. Our point of view follows the one of multifield theories /9-12/ that seem to be an adequate tool in investigating the intricate nature of complex materials and unifying common special models in material sciences, in particular of soft condensed matter.

For any manifold $M$, we indicate by $\mathrm{T}_{\mathrm{m}} M$ its tangent space at $\mathrm{m} \in M$ and by $\mathrm{T} M$ its tangent bundle.

\section{A BRIEF SKETCH OF SPATIAL THERMOMECHANICS OF COMPLEX BODIES}

To manage a framework applicable directly to solids and fluids (or to the combination of them), we sketch here the basic balance equations of the thermomechanics of complex bodies in a pure spatial description free of any concept of reference place or paragon settings and without even formal (and rather artificial) recourse to an inverse motion. In this way we follow a point of view presented in $/ 9 /$ within an isothermal Hamiltonian variational framework and develop it in part towards non-isothermal issues.

For us $E$ and $V e c$ denote respectively the three-dimensional Euclidean point space and its associated translation space. $\mathrm{B}$ is a regular region of $E$ with surface-like boundary where the normal is well defined everywhere except at a finite number of corners and edges. Each $x \in B$ is the place of a material element "collapsed" ideally to a point in the standard practice of continuum mechanics. However, in complex bodies, 
the material element has its own substructure; it is a "system" and the geometrical picture restricted to its sole place in space is too simplicistic, and we then need to introduce a morphological descriptor of the material substructure at least at a coarse grained level. By following the primary setting of multifield theories $/ 9-12 /$, we define a field

$$
\mathrm{B} \ni \mathrm{x} \rightarrow \mathrm{v}(\mathrm{x}) \in M,
$$

where $M$ is a finite-dimensional differentiable paracompact manifold without boundary. We do not specify the nature of $v$ to encompass a class of substructures as large as possible, however it will be evident that some properties associated with thermodynamic stability can be investigated only by adding further geometrical structures to $M$. In the meantime, we remark that, in general, $M$ is not a linear space (as in most special cases of prominent physical interest), and this is the major source of difficulties additional to the common ones of continuum mechanics.

In a time interval $\left[0, t^{*}\right]$, a spatial velocity field

$$
B \times\left[0, t^{*}\right] \rightarrow(x, t) \rightarrow v(x, t) \in V e c
$$

describes the "departure" from B during a motion characterized also by changes in its substructural state, namely

$$
\left[0, t^{*}\right] \rightarrow t \rightarrow v(x, t) \in M
$$

Changes in substructural states are of various nature. The ones associated with rigid changes of observers (where different observers differing by rigid motions may evaluate different values of $v$, i.e., different representations of $M$ ) have prominent importance. Let $\dot{q} \wedge$ (a skew symmetric tensor, where $\dot{q}$ is the rotational velocity), be an element of the tangent space $S O(3)$ at the identity, we indicate by $A \dot{q}$ its action at $v$, and interpret $\dot{v}_{R}=A \dot{q}$ as a special "rigid" change of the substructural state.

The geometrical picture is completed by the spatial metrics $\mathrm{g}(\mathrm{x})$ on $E$, i.e.,

$$
\mathbf{x} \rightarrow \mathbf{g}(\mathbf{x}) \in \operatorname{Sym}^{+}(V e c, V e c)
$$

During motion, standard and substructural interactions of bulk and contact nature occur and need to be balanced.

Bulk interactions are

- standard forces $b$, such that $B \rightarrow x \rightarrow b(x) \in T_{x}{ }^{*} B$,

- substructural ones $\beta$, such that $B \rightarrow x \rightarrow \beta(x) \in T_{x}{ }^{*} B$, both including inertial effects. 
Contact interactions are

- Cauchy stress $\mathrm{T}$, such that $\mathrm{B} \ni \mathrm{x} \rightarrow \mathrm{T}(\mathrm{x}) \in \operatorname{Hom}\left(V e c, \mathrm{~T}_{\mathrm{x}}{ }^{*} \mathrm{~B}\right)$,

- microstress $\mathrm{S}$, such that $\mathrm{B} \ni \mathrm{x} \rightarrow \mathrm{S}(\mathrm{x}) \in \operatorname{Hom}\left(\operatorname{Vec}, \mathrm{T}_{\mathrm{v}(\mathrm{x})}{ }^{*} M\right)$.

They satisfy the following integral balances holding on any regular subset $b$ of non negligible volume B:

$$
\begin{aligned}
& \int_{b} \mathrm{~b}+\int_{\partial b} \mathrm{Tn}=0, \\
& \int_{b}\left(\left(\mathrm{x}-\mathrm{x}_{0}\right) \wedge \mathrm{b}+A^{\mathrm{T}} \beta\right)+\int_{\partial b}\left(\left(\mathrm{x}-\mathrm{x}_{0}\right) \wedge \mathrm{T} \mathrm{n}+A^{\mathrm{T}} \mathrm{Sn}\right)=0,
\end{aligned}
$$

where $\mathrm{n}$ is the outward unit normal to the surface $\partial b$ (the boundary of $b$ ) and $A$, defined above, is a linear operator mapping at each $x$ spatial vectors into elements of $\mathrm{T}_{v(\mathbf{x})} M$.

The arbitrariness of $b$ implies the local Cauchy balance of standard interactions

$$
b+\operatorname{div} T=0
$$

while, from the non-standard balance of moments (6), we obtain

$$
\mathrm{eT}=A^{\mathrm{T}}(\beta+\operatorname{div} \mathrm{S})+\left(\operatorname{grad} A^{\mathrm{T}}\right) \mathrm{S}
$$

where $\mathrm{e}$ is the Ricci's indicator.

However, since $A$ is not one-to-one by definition, there exists $\mathrm{z}$ such that $\mathrm{B} \ni \mathrm{x} \rightarrow \mathrm{z}(\mathrm{x}) \in T_{v(x)}{ }^{*} M$ satisfying

$$
A^{\mathrm{T}} \mathrm{z}=\mathrm{eT}-\left(\operatorname{grad} A^{\mathrm{T}}\right) \mathrm{S}
$$

$\operatorname{div} S-z+\beta=0$.

The identification of inertial effects is simply obtained by starting from the additive decomposition into inertial and non-inertial parts, namely $b=b^{\text {in }}+b^{\text {ni }}, \beta=\beta^{\text {in }}+\beta^{\text {ni }}$, and using the integral balance

$$
\int_{b}\left((1 / 2 \rho v \cdot v+k(v, \dot{v}))+j_{b}\left(b^{\text {in }} \cdot v+\beta^{\text {in }} \cdot \dot{v}\right)=0\right.
$$

where $\mathbf{k}(v, \dot{v})$ is the possible kinetic energy pertaining to the substructure. In writing (11), we require imp!icitly that $v(x, t)$ be kinematically compatible; in other words, for $t^{*}>t$ we require the existence of 
sufficiently smooth maps $B \times\left[0, t^{*}\right] \ni(x, t) \rightarrow y(x, t) \in E$ being, at each $t$, injective and orientation preserving, such that at each $x$ one has $(d / d t)(y(x, t))=v(x, t)$.

From (11), based to the arbitrariness of $b, v$ and $v$, we get the standard identification [10]

$$
\mathbf{b}^{\text {in }}=-\rho \dot{v}, \quad \beta^{\text {in }}=d / d t\left(\partial_{\dot{v}} \chi\right)-\partial_{v} \chi,
$$

where $\chi(v, \dot{v})$ is such that $k(v, \dot{v})=\partial_{\dot{v}} \chi-\chi$ and is called kinetic co-energy.

We complete our sketch by recalling the first and second law of thermodynamics, once densities $(x, t) \rightarrow$ $\varepsilon(x, t)$ and $(x, t) \rightarrow \eta(x, t)$ of internal energy and entropy, respectively, are introduced. We can write

$$
\begin{aligned}
& (\mathrm{d} / \mathrm{dt})\left(\int_{b} \varepsilon\right)+\int_{b}(\mathrm{~T} \cdot \operatorname{grad} \mathbf{v}+\mathbf{z} \cdot \dot{v}+\mathrm{S} \cdot \operatorname{grad} \dot{v})+\int_{\partial b} \mathbf{q} \cdot \mathbf{n}=\mathbf{0}, \\
& (\mathrm{d} / \mathrm{dt})\left(\int_{b} \eta\right)+\int_{\partial b} \mathbf{h} \cdot \mathbf{n}<0,
\end{aligned}
$$

where $q$ and $h$ respectively are the fluxes of heat and entropy related by $h=q / \theta$, with $\theta$ the absolute temperature. We do not consider heat and entropy sources for the sake of simplicity.

We remark that, if we presume that the measures of interaction admit constitutive structures of the form

$$
T=T(g, \theta, v, \operatorname{grad} v), \quad z=z(g, \theta, v, \operatorname{grad} v), \quad S=S(g, \theta, v, \operatorname{grad} v)
$$

and introduce the free energy $\psi=\varepsilon-\theta \eta$, presuming that

$$
\psi=\psi(\mathrm{g}, \theta, v, \operatorname{grad} v)
$$

By using Clausius-Duhem inequality simply obtained from (13) and (14), we get

$$
\mathrm{T}=2 \partial_{\mathrm{g}} \psi \mathrm{g}-(\operatorname{grad} v) \mathrm{S}, \quad \mathrm{z}=\partial_{v} \psi, \quad \mathrm{S}=\partial_{\mathrm{grad} v} \psi
$$

Note that (17a) is a natural extension of the Doyle-Eriksen formula.

We remark here that, if $\theta_{c}$ is the environmental temperature, an appropriate version of the Eriksen's lemma holds and one finds the existence of a function

$$
\mathrm{\kappa}: \mathrm{B} \times S y m^{+} \times \mathrm{R}^{++} \times M \times \operatorname{Hom}(V e c, \mathrm{~T} M) \rightarrow \mathrm{R}
$$

such that

$$
\psi(\mathrm{g}, \theta, v, \operatorname{grad} v)=\varepsilon(\mathrm{g}, \theta, v, \operatorname{grad} v)-\theta_{\mathrm{e}} \eta(\mathrm{g}, \theta, v, \operatorname{grad} v)=
$$




$$
=\psi\left(\mathrm{g}, \theta_{\mathrm{e}}, v, \operatorname{grad} v\right)+\kappa(\mathrm{g}, \theta, v, \operatorname{grad} v)\left(\theta-\theta_{e}\right)^{2}
$$

where $\kappa(x, g, \theta, v, \operatorname{grad} v)>0$.

\section{STATES, TOPOLOGY IN THE STATE-SPACE AND A LIAPUNOV FUNCTION}

For us a state is the list $(\mathrm{g}, \theta, \mathrm{v}, \mathrm{v}, \dot{\mathrm{v}})$. We indicate it by $\sigma$ and call $\Sigma$ the collection of possible $\sigma$ 's for a given body. We call stationary states $\sigma_{\mathrm{s}}$ that of the form $(\mathrm{g}, \theta, 0, v, 0)$.

The topology over $\Sigma$ is the product topology induced by the topologies over the spaces in which the elements of $\sigma$ live. A basic difficulty is given by the presence of the mapping $v$, because it takes values over a non-linear manifold. If $M$ is Riemannian, we have a natural distance $\mathrm{d}_{M}\left(v_{1}(\mathrm{x}), v_{2}(\mathrm{x})\right)$ over it, between two arbitrary points $v_{1}(x)$ and $v_{2}(x)$, calculated locally by making use of the geodetics over $M$. In particular, since $d_{M}$ can be unbounded, to assure boundedness, we adopt a scaled metrics $d_{M}{ }^{*}=d_{M} /\left(1+d_{M}\right)$ and define

$$
\mathrm{d}\left(v_{1}, v_{2}\right)=\int_{B} d_{M} \cdot\left(v_{1}(x), v_{2}(x)\right)
$$

following in this way the procedure introduced in $/ 12 /$, where the construction of other possible (physically significant) metrics is also discussed.

To define distances between rate fields of the order parameter, we need to add further structure over $M$, namely a parallelism and a scalar product $\left\langle\cdot, \cdot>\right.$ in each $T_{v(x)}{ }^{*} M$. More precisely, at each $x$, the pair $(v(x)$, $\dot{v}(\mathrm{x}))$ is an element of the tangent space of $M$ and cannot be decomposed invariantly unless we have a parallelism. The scalar product allows also the introduction of an $\mathrm{L}^{2}$ norm of the type (see /12/)

$$
\begin{aligned}
& \left\|\dot{v}_{1}-\dot{v}_{2}\right\|_{\left.L^{2}(B, T M)\right)}=(1 / 2)\left(\int_{B}<\dot{v}_{1}(x)-\dot{v}_{2}^{*}(x), \quad \dot{v}_{1}(x)-\dot{v}_{2}^{*}(x)>\right)^{1 / 2}+ \\
& +(1 / 2)\left(\int_{B}<\dot{v}_{1}^{*}(x)-\dot{v}_{2}(x), \quad \dot{v}_{1}(x)-\dot{v}_{2}(x)>\right)^{1 / 2}
\end{aligned}
$$

Note the difference $\dot{v}_{1}-\dot{v}_{2} *$ makes sense for two reasons: (i) the existence of a parallelism allows us to transport $\dot{v}_{2}(x)$ from $\mathrm{T}_{v_{2}(x)} M$ to $\mathrm{T}_{v_{1}(\mathrm{x})} M$, where it becomes $\dot{v}_{2}{ }^{*}(\mathrm{x})$ (ii) each $\mathrm{T}_{\mathrm{v}(\mathrm{x})} M$ is a linear space. An analogous meaning has the difference $\dot{v}_{1}^{*}(x)-\dot{v}_{2}(x)$; in fact, $\dot{v}_{1}(x)$ is transported from $T_{v_{1}}(x) M$ to $\mathrm{T}_{\mathrm{v}_{2}(\mathrm{x})} M$, where it becomes $\dot{\mathrm{v}}_{1}{ }^{*}(\mathrm{x})$.

With analogous meaning of symbols we define (taking the formula from $/ 12 /)$

$$
\begin{aligned}
& \left\|\operatorname{grad} v_{1}-\operatorname{grad} v_{2}\right\|_{L^{2}(T B . T M)}=(1 / 2)\left(\int_{B}<\operatorname{grad} v_{1}(\mathrm{x})-\operatorname{grad} v_{2}^{*}(\mathbf{x}), \operatorname{grad} v_{1}(\mathbf{x})-\operatorname{grad} v_{2}^{*}(\mathbf{x})>^{\circ}\right)^{1 / 2} \\
& +(1 / 2)\left(\int_{B}<\operatorname{grad} v_{1}^{*}(\mathbf{x})-\operatorname{grad} v_{2}(x), \operatorname{grad} v_{1}^{*}(\mathbf{x})-\operatorname{grad} v_{2}(x)>^{0}\right)^{1 / 2}
\end{aligned}
$$


where $\left\langle, \cdot>^{\circ}\right.$ is a scalar product over $\mathrm{Hom}\left(\mathrm{Vec}, \mathrm{T}_{(\mathbf{x})}{ }^{\circ} \mathrm{M}\right)$ at each $\mathbf{x}$.

Finally, we endow the state space $\Sigma$ with a distance $d(\cdot, \cdot)$ given by

$$
\begin{aligned}
& d\left(\sigma_{1}, \sigma_{2}\right)=\left\|v_{1}-v_{2}\right\|_{L^{2}(B ~ V e c)}+\left\|g_{1}-g_{2}\right\|_{L^{2}(B, S y m)}+ \\
& +d\left(v_{1}, v_{2}\right)+\left\|\dot{v}_{1}-\dot{v}_{2}\right\|_{L^{2}(B \text { TM })}+\left\|\nabla v_{1}-\nabla v_{2}\right\|_{L^{2}(T B T M)}
\end{aligned}
$$

Let $\Omega$ be an open subset of $\Sigma$. Let also $\mathrm{E}(\sigma)$ be the total energy given by the integral over B of the free energy, plus the potential of external forces calculated at $\theta=\theta_{\mathrm{e}}$. If $\Omega$ contains a stationary state $\sigma_{0}$ such that (1) E has a minimum at $\sigma_{0}$ over $\Omega$, and (2) inf $\{\mathrm{E}(\sigma), \sigma \in \partial \Omega\}>\mathrm{E}\left(\sigma_{0}\right)$, then $\sigma_{0}$ is called an equilibrium state and $\Omega$ a potential wall.

We indicate by $\Sigma_{0}^{m}$ the set of equilibrium states and denote with $E q u i l(\sigma)$ the mapping Equil $: \Sigma \rightarrow \Sigma$ associating at each state a corresponding equilibrium value. Here we follow $/ 4 /$; however, on the contrary to /4/, we imagine that Equil can associate two or more equilibrium states to each $\sigma$ when there is a possible frustration.

Let us now define

$$
V(\sigma)=U(\sigma)+\int_{B}\left(\psi\left(g, \theta_{e}, v, \operatorname{grad} v\right)+\kappa\left(g, \theta_{e}, v, \operatorname{grad} v\right)\left(\theta-\theta_{e}\right)+1 / 2 \rho v \cdot v+k(v, v)\right)
$$

\section{Theorem 1}

For any $\sigma$ there exists an open neighborood $\Omega(\sigma)$ such that

(1) $(\mathrm{V}-\mathrm{VoEquil})(\sigma)>0$

(2) the equality sign holds if and only if $\sigma \in \Sigma^{\mathrm{eq}}$.

If we consider a path $t \rightarrow \sigma(t)$ in the state space, we have consequent time variations $t \rightarrow(v(t), k(t), k(t)$, $E(t))$.

\section{Theorem 2}

E has a global minimum at $\sigma_{0}$, then given $\varepsilon>0$ there exists $\delta>0$ such that any path $\left[0, t^{*}\right] \ni t \rightarrow \sigma(t) \in \Sigma$ with

$$
\begin{aligned}
& \int_{B}(v \cdot v)(0)<\delta, \quad \int_{B} k(0)\left(\theta(0)-\theta_{C}\right)^{2}<\delta, \\
& \int_{B} k(0)<\delta, \quad E(0)-E\left(\sigma_{0}\right)<\delta,
\end{aligned}
$$

satisfies

$$
\begin{aligned}
& \int_{B}(v \cdot v)(\mathrm{t})<\varepsilon, \quad \int_{B} \kappa(\mathrm{t})\left(\theta(\mathrm{t})-\theta_{\mathrm{e}}\right)^{2}<\varepsilon, \\
& \int_{B} \mathrm{k}(\mathrm{t})<\varepsilon, \quad \mathrm{E}(\mathrm{t})-\mathrm{E}\left(\sigma_{0}\right)<\varepsilon, \\
& -\int_{0}{ }^{*} \int_{B}\left(1 /\left(\rho \theta^{2}\right)\right)(\mathrm{q}(\mathrm{F}, \theta, \operatorname{grad} \theta, v, \operatorname{grad} v) \cdot \operatorname{grad} \theta)<\varepsilon .
\end{aligned}
$$


Such a theorem extends to complex bodies the analogous result of $/ 4 /$.

For compressible complex fluids, the representation of the state is more simple and we have $\sigma=(u, \theta, v, v, \dot{v})$, where $u=\operatorname{det}(\mathrm{g})$.

\section{REFERENCES}

1. J.L. Ericksen, A thermo-kinetic view of elastic stability theory, International Journal of Solids and Structures, 2, 573-580 (1966).

2. B.D. Coleman and J.M. Greenberg, Thermüynamics and stability of fluid motion, Archive for Rational Mechanics and Analysis, 25, $321-341$ (1967).

3. B.D. Coleman, On the stability of equilibrium states of general fluids, Archive for Rational Mechanics and Analysis, 36, 1-32 (1970).

4. M.E. Gurtin, Thermodynamics and stability, Archive for Rational Mechanics and Analysis, 59, 63-96 (1975).

5. J.R. Dunwoody and R.W. Ogden, On the thermodynamic stability of elastic heat-conducting solids subjected to a deformation-temperature constraint, Mathematics and Mechanics of Solids, 7, 285-306 (2002).

6. M. Shainpoor and G. Ahmadi, Stability of Cosserat fluid motion, Archive for Rational Mechanics and Analysis, 47, 188-194 (1972).

7. F. Bethuel, H. Brezis, B.D. Coleman and F. Hélein, Bifurcation analysis of minimizing harmonic maps describing the equilibrium of nematic phases between cylinders, Archive for Rational Mechanics and Analysis, 118, 149-168 (1992).

8. J.D. Goddard, Material instability in complex fluids, Annual Review of Fluid Dynamics, 35, 113-133 (2003).

9. G. Capriz and P.M. Mariano, Symmetries and Hamiltonian formalism for complex materials, $J$ ، Elasticity, 72, 57-70, (2003).

10. G. Capriz, Continua with Microstructure, Springer-Verlag, Berlin (1989).

11. P.M. Mariano, Multifield theories in mechanics of solids, Advances in Applied Mechanics, 38, 1-93 (2002).

12. C. de Fabritiis and P.M. Mariano, Geometry of interactions in complex bodies, Journal of Geometry and Physics, 54, $301-323$ (2005). 
